\title{
Naturalness and lepton number/flavor violation in inverse seesaw models
}

\author{
Naoyuki Haba, ${ }^{a}$ Hiroyuki Ishida $^{a, b}$ and Yuya Yamaguchi ${ }^{a, c}$ \\ ${ }^{a}$ Graduate School of Science and Engineering, Shimane University, \\ 1060, Nishikawatsu, Matsue, Shimane, Japan \\ ${ }^{b}$ Physics Division, National Center for Theoretical Sciences, \\ 101, Section 2 Kuang Fu Road, Hsinchu, 300 Taiwan \\ ${ }^{c}$ Department of Physics, Faculty of Science, Hokkaido University, \\ Kita 9 Nishi 8, Kita-ku, Sapporo, Hokkaido, Japan \\ E-mail: haba@riko.shimane-u.ac.jp, hiroyuki403@gmail.com, \\ yy@particle.sci.hokudai.ac.jp
}

ABSTRACT: We introduce three right-handed neutrinos and three sterile neutrinos, and consider an inverse seesaw mechanism for neutrino mass generation. From naturalness point of view, their Majorana masses should be small, while it induces a large neutrino Yukawa coupling. Then, a neutrinoless double beta decay rate can be enhanced, and a sizable Higgs mass correction is inevitable. We find that the enhancement rate can be more than ten times compared with a standard prediction from light neutrino contribution alone, and an analytic form of heavy neutrino contributions to the Higgs mass correction. In addition, we numerically analyze the model, and find almost all parameter space of the model can be complementarily searched by future experiments of neutrinoless double beta decay and $\mu \rightarrow e$ conversion.

Keywords: Neutrino Physics, Beyond Standard Model

ARXIV EPRINT: 1608.07447 


\section{Contents}

1 Introduction 1

2 Mass spectrum in inverse seesaw models 2

3 Neutrinoless double beta decay $\quad 5$

$\begin{array}{lll}4 & \text { Higgs mass correction } & 7\end{array}$

$\begin{array}{llr}5 & \text { Numerical analysis } & 8\end{array}$

$\begin{array}{lll}6 & \text { Summary } & 13\end{array}$

$\begin{array}{ll}\text { A } \mu \rightarrow e \text { conversion rate } & 13\end{array}$

\section{Introduction}

The experimental results on the neutrino oscillation have established an exact evidence of neutrino masses. Since the origin of neutrino masses cannot be explained within the standard model (SM), there are a lot of models towards explaining the tiny neutrino masses naturally. The type-I seesaw model [1-6] is one of the simplest idea, in which right-handed neutrinos are introduced. There is a large parameter space for their masses, and they could be solve some phenomenological problems: short baseline neutrino oscillation anomalies with a eV mass, ${ }^{1}$ relic abundance of dark matter with a keV mass, and baryon asymmetry of the universe (BAU) with $\mathrm{GeV}$ to $\mathrm{TeV}$ masses with a sufficient fine-tuning [see ref. [16] for a review].

In the conventional type-I seesaw model, the tiny neutrino masses and the BAU can be simultaneously explained without a fine-tuning, in which the right-handed neutrinos are typically heavier than $10^{9} \mathrm{GeV}$ [17]. It is, however, impossible to search such heavy right-handed neutrinos directly. For the indirect searches, since the Majorana masses of right-handed neutrinos violate the lepton number conservation, lepton number violation processes might be detectable. Nevertheless, the absence of the neutrinoless double beta decay at the moment [18] suggests the approximate lepton number conservation. Then, the Majorana masses should be small from the naturalness in the sense of 't Hooft [19].

On the other hand, inverse seesaw models [20-22] are much interesting from experimental point of view. In the inverse seesaw models, there are right-handed neutrinos which couple with left-handed neutrinos and sterile neutrinos which do not couple with lefthanded neutrinos. Compared with the type-I seesaw model, their Majorana masses can be

\footnotetext{
${ }^{1}$ The neutrino oscillation anomalies have been reported by some experiments: reactor [7-9], accelerator [10-13] and Gallium [14, 15].
} 
smaller, which is preferred by the naturalness, and sizable neutrino Yukawa couplings are allowed. Thus, the inverse seesaw model has been strongly constrained by lepton flavor violations [23], cosmology [24] and collider experiments [25-27]. Moreover, the inverse seesaw model can be highly testable by precise measurements of lepton flavor violation (for example, $\mu \rightarrow e \gamma$ [28], $\mu \rightarrow$ eee [29], and $\mu \rightarrow e$ conversion [30]), and high energy/luminosity collider experiments [31-42].

In this paper, we focus on the $(3,3)$ inverse seesaw model, in which the number of both right-handed neutrinos and sterile neutrinos are three, and we do not consider the phenomenological issues mentioned above.

This paper is organized as follows. In section 2, we briefly review the inverse seesaw mechanism and define our setup. In section 3, we investigate heavy neutrino contributions for the neutrinoless double beta decay, and find an analytic form of the heavy neutrino contribution to the effective neutrino mass. In section 4, we also investigate the Higgs mass correction coming from heavy neutrinos, which should be not so larger than the electroweak (EW) scale from the naturalness point of view. In section 5, we show numerical results of heavy neutrino contributions to the effective neutrino mass and the Higgs mass correction. Finally, we summarize our results in section 6.

\section{Mass spectrum in inverse seesaw models}

In this section, we explain a mass spectrum in inverse seesaw models. To realize the inverse seesaw mechanism, we introduce three pairs of SM gauge singlet fields $(N, S)$, which have the lepton number $L=1$. Then, the Lagrangian contains

$$
\mathcal{L}=i \bar{N} \gamma^{\mu} \partial_{\mu} N+i \bar{S} \gamma^{\mu} \partial_{\mu} S-M_{N} \bar{N} S-\frac{\mu_{N}}{2} \overline{N^{c}} N-\frac{\mu_{S}}{2} \overline{S^{c}} S-Y_{\nu} \bar{N} \tilde{\Phi} \ell+\text { h.c. },
$$

where $\ell$ is the lepton doublet, which has the lepton number $L=1$, and $\tilde{\Phi}=i \sigma_{2} \Phi$ is the charge conjugation of the Higgs doublet $\Phi$. The $3 \times 3$ complex matrices $M_{N}, \mu_{N, S}$, and $Y_{\nu}$ stand for Dirac-type mass, Majorana masses, and neutrino Yukawa coupling, respectively. We have assumed that $S$ does not couple with $\ell$ at tree level, ${ }^{2}$ and will call $N$ and $S$ right-handed neutrino and sterile neutrino, respectively.

After the EW symmetry breaking, the Higgs obtains a nonzero vacuum expectation value, $\langle\Phi\rangle=v / \sqrt{2} \simeq 174 \mathrm{GeV}$, and then, the Dirac neutrino mass matrix is induced by $m_{D}=Y_{\nu}\langle\Phi\rangle$. In the basis of $n_{R}=\left(\nu_{L}^{c}, N, S\right)^{T}$, the neutrino mass terms become

$$
\mathcal{L}_{\text {mass }}=-\frac{1}{2} n_{R}^{T} C \mathcal{M} n_{R}+\text { h.c. }, \quad \text { with } \quad C=i \gamma^{2} \gamma^{0},
$$

where the $9 \times 9$ neutrino mass matrix $\mathcal{M}$ is given by

$$
\mathcal{M}=\left(\begin{array}{ccc}
0 & m_{D}^{T} & 0 \\
m_{D} & \mu_{N} & M_{N} \\
0 & M_{N}^{T} & \mu_{S}
\end{array}\right) .
$$

\footnotetext{
${ }^{2}$ In ref. [43], the authors introduced vector-like gauge-singlet fermions, and denoted that Yukawa coupling of the one field, which corresponds to $S$ in our notation, can be always removed rotating vector-like gaugesinglet fermions.
} 
The lepton number is broken by two Majorana masses $\mu_{N}$ and $\mu_{S}$, and they should be much smaller than $M_{N}$ and $m_{D}$ in the 't Hooft's sense of naturalness. In the technically natural limit of $\mu_{N, S} \ll m_{D}, M_{N}$, the order of magnitude of mass eigenvalues are given by

$$
\begin{aligned}
\mathcal{O}\left(m_{\nu}\right) & =\frac{m_{D}^{2} \mu_{S}}{m_{D}^{2}+M_{N}^{2}}+\mathcal{O}\left(\mu_{N, S}^{2}\right), \\
\mathcal{O}\left(m_{ \pm}\right) & = \pm \sqrt{m_{D}^{2}+M_{N}^{2}}+\frac{M_{N}^{2} \mu_{S}}{2\left(m_{D}^{2}+M_{N}^{2}\right)}+\frac{\mu_{N}}{2}+\mathcal{O}\left(\mu_{N, S}^{2}\right) .
\end{aligned}
$$

In the limit of $\mu_{N, S} \rightarrow 0$, active neutrinos become massless and lepton number conservation is restored. Note that the active neutrino mass $m_{\nu}$ can be suppressed by small $\mu_{S}$, but does not depend on $\mu_{N}$ at the tree-level. ${ }^{3}$ For $m_{D} \ll M_{N}$, the heavy neutrino masses are approximated by $\mathcal{O}\left(m_{ \pm}\right) \simeq \pm M_{N}+\left(\mu_{S}+\mu_{N}\right) / 2$, and thus, the mass difference between them is small as $\mathcal{O}\left(\mu_{N, S}\right)$.

To explain the active neutrino mass scale $m_{\nu} \sim 0.1 \mathrm{eV}$, if there is no accidental cancellation, or no fine-tuning, the energy scale of $\mu_{S}$ should be

$$
\mu_{S} \sim\left(\frac{M_{N}}{1 \mathrm{TeV}}\right)^{2}\left(\frac{1}{Y_{\nu}}\right)^{2} \times 1 \mathrm{eV},
$$

for a given $M_{N}$ and $Y_{\nu}$. Technically natural limit of $\mu_{N, S} \ll m_{D}, M_{N}$ requires

$$
0.1 \mathrm{eV} \lesssim \mu_{S} \ll 100 \mathrm{GeV}
$$

where the lower and upper bounds have been obtained by $m_{\nu} \sim \mu_{S}\left(m_{D} / M_{N}\right)^{2} \lesssim \mu_{S}$, and $m_{D} \lesssim 100 \mathrm{GeV}$ corresponding to a perturbativity bound $Y_{\nu} \lesssim 1$, respectively. Using eq. (2.6) and $Y_{\nu} \lesssim 1, M_{N} \ll 10^{8} \mathrm{GeV}$ is required to satisfy eq. (2.7). Actually, $\mu_{S}$ and $M_{N}$ are constrained more severely by the lepton flavor violation as we will show in section 5 .

In the matrix form, active neutrino mass matrix is given by

$$
\begin{aligned}
m_{\nu} & \simeq m_{D}^{T}\left(M_{N}^{T}\right)^{-1} \mu_{S} M_{N}^{-1} m_{D} \\
& =m_{D}^{T} X^{-1} m_{D}
\end{aligned}
$$

where we have defined $X=M_{N} \mu_{S}^{-1} M_{N}^{T}$, and the last form is the same as the type-I seesaw model [1-6]. The active neutrino mass matrix is diagonalized by Pontecorvo-MakiNakagawa-Sakata (PMNS) matrix $U_{\text {PMNS }}[47,48]$ :

$$
U_{\mathrm{PMNS}}^{T} m_{\nu} U_{\mathrm{PMNS}}=\operatorname{diag}\left(m_{1}, m_{2}, m_{3}\right) \equiv D_{m}
$$

where $m_{i}(i=1,2$, and 3$)$ are the mass eigenvalues of three lightest neutrinos. Actually, since this diagonalization is satisfied at leading order level, we will define an unitary matrix diagonalizing the full $9 \times 9$ neutrino mass matrix in the end of this section, and use it for numerical calculations.

\footnotetext{
${ }^{3}$ There are one-loop corrections induced by the $Z$ and Higgs bosons, which give a contribution proportional to $\mu_{N}$, and they could be larger than $0.1 \mathrm{eV}$ [44-46]. We have checked that our numerical results shown in section 5 avoid such large one-loop corrections.
} 
Using the Casas-Ibarra parametrization [49], the neutrino Yukawa coupling matrix is parametrized by

$$
Y_{\nu}=\frac{\sqrt{2}}{v} V^{\dagger} \sqrt{D_{X}} R \sqrt{D_{m}} U_{\mathrm{PMNS}}^{\dagger}
$$

where $V$ is a unitary matrix, which diagonalizes $X$ by $V X V^{T}=\operatorname{diag}\left(X_{1}, X_{2}, X_{3}\right) \equiv D_{X}$. The complex orthogonal matrix $R$ can be parameterized by

$$
R=\zeta\left(\begin{array}{ccc}
c_{12} c_{13} & s_{12} c_{13} & s_{13} \\
-s_{12} c_{23}-c_{12} s_{23} s_{13} & c_{12} c_{23}-s_{12} s_{23} s_{13} & s_{23} c_{13} \\
s_{12} s_{23}-c_{12} c_{23} s_{13} & -c_{12} s_{23}-s_{12} c_{23} s_{13} & c_{23} c_{13}
\end{array}\right)
$$

where $c_{i j}=\cos \omega_{i j}$ and $s_{i j}=\sin \omega_{i j}$ with arbitrary complex angles $\omega_{i j}$. The overall sign $\zeta= \pm \mathbf{1}$ ( $\mathbf{1}$ is an $3 \times 3$ identity matrix) corresponds to degree of freedom of a parity transformation, which determines $\operatorname{det}[R]= \pm 1$ for $\zeta= \pm \mathbf{1}$. In the following, we assume that $M_{N}, \mu_{S}$ and $\mu_{N}$ are diagonal, and also $\mu_{N}=\mu_{S}$ for simplicity: $M_{N}=D_{M} \equiv \operatorname{diag}\left(M_{1}, M_{2}, M_{3}\right)$ and $\mu_{N}=\mu_{S}=D_{\mu} \equiv \operatorname{diag}\left(\mu_{1}, \mu_{2}, \mu_{3}\right)$. Then, $X=$ $\operatorname{diag}\left(X_{1}, X_{2}, X_{3}\right)=\operatorname{diag}\left(M_{1}^{2} / \mu_{1}, M_{2}^{2} / \mu_{2}, M_{3}^{2} / \mu_{3}\right)$, and $V$ becomes a unit matrix.

When both $M_{N}$ and $\mu_{N, S}$ are diagonal, before the EW symmetry breaking, mass eigenvalues of heavy neutrinos become

$$
m_{i \pm}= \pm M_{i}+\mu_{i} \quad \text { for } \quad i=1,2, \text { and } 3,
$$

because of $m_{D}=0$. The mass eigenvectors are given by $N_{i \pm}=\left(N_{i} \pm S_{i}\right) / \sqrt{2}$, and they have the same neutrino Yukawa couplings like $\left(Y_{\nu} / \sqrt{2}\right) \overline{N_{i \pm}} \tilde{\Phi}^{\dagger} \ell$. Therefore, before the EW symmetry breaking, there exist the almost identical particles, which have the same couplings and masses with a small difference $\mu_{i}$.

Since the degenerate heavy neutrinos naturally arise in inverse seesaw models, the BAU can be explained by leptogenesis through the neutrino oscillation [50, 51]. On the other hand, if the number of sterile neutrinos are larger than the number of right-handed neutrinos, there exit additional mass eigenstates with their masses of $\mathcal{O}\left(\mu_{S}\right)$. The new mass eigenstates can explain the short baseline neutrino oscillation anomalies with $\mu_{S} \sim$ $\mathcal{O}(\mathrm{eV})$ [52], and/or the relic density of dark matter with $\mu_{S} \sim \mathcal{O}(\mathrm{keV})$ [53]. However, these issues are beyond the scope of this paper.

For the following discussion, it is useful to show the mass matrix in the $\left(\nu_{L}^{c}, N_{i \pm}\right)^{T}$ basis:

$$
\mathcal{M}=\left(\begin{array}{ccccccc}
0_{3 \times 3} & \left(m_{D}^{\prime T}\right)_{\alpha 1} & \left(m_{D}^{\prime T}\right)_{\alpha 1} & \left(m_{D}^{\prime T}\right)_{\alpha 2} & \left(m_{D}^{\prime T}\right)_{\alpha 2} & \left(m_{D}^{\prime T}\right)_{\alpha 3} & \left(m_{D}^{\prime T}\right)_{\alpha 3} \\
\left(m_{D}^{\prime}\right)_{1 \alpha} & m_{1-} & 0 & 0 & 0 & 0 & 0 \\
\left(m_{D}^{\prime}\right)_{1 \alpha} & 0 & m_{1+} & 0 & 0 & 0 & 0 \\
\left(m_{D}^{\prime}\right)_{2 \alpha} & 0 & 0 & m_{2-} & 0 & 0 & 0 \\
\left(m_{D}^{\prime}\right)_{2 \alpha} & 0 & 0 & 0 & m_{2+} & 0 & 0 \\
\left(m_{D}^{\prime}\right)_{3 \alpha} & 0 & 0 & 0 & 0 & m_{3-} & 0 \\
\left(m_{D}^{\prime}\right)_{3 \alpha} & 0 & 0 & 0 & 0 & 0 & m_{3+}
\end{array}\right)
$$


where $m_{D}^{\prime}=m_{D} / \sqrt{2}$. Now, we define $U$ as an unitary matrix diagonalizing this full $9 \times 9$ mass matrix, which is given in the following form:

$$
U^{T} \mathcal{M} U=\mathcal{M}_{\text {diag }} \quad \text { with } \quad\left(\nu_{e}, \nu_{\mu}, \nu_{\tau}, N_{1-}, N_{1+}, N_{2-}, N_{2+}, N_{3-}, N_{3+}\right)^{T}=U_{\alpha i} \nu_{i} .
$$

The mass eigenstates $\nu_{1}, \nu_{2}$ and $\nu_{3}$ correspond to the active neutrinos, and $\nu_{i}$ 's $(i=4 \sim 9)$ are the heavy neutrinos in light order from $i=4$ to 9 . For $m_{D} \ll M_{N}$, the heavy mass eigenstates are almost composed of $N_{i \pm}$. In particular, diagonal elements $U_{i i}(i=4 \sim 9)$ are nearly unity, unless $M_{N}$ is degenerate. In addition, matrix elements expressing the leftright mixing are almost satisfy $U_{\alpha 4}^{2} \simeq U_{\alpha 5}^{2}, U_{\alpha 6}^{2} \simeq U_{\alpha 7}^{2}$ and $U_{\alpha 8}^{2} \simeq U_{\alpha 9}^{2}$ for $\alpha=e, \mu$ and $\tau$, which are exactly satisfied in the limit of $\mu_{i} \rightarrow 0$.

\section{Neutrinoless double beta decay}

The massive Majorana neutrinos induce neutrinoless double beta decay, and its rate is proportional to the squared of the effective neutrino mass, which is given by

$$
m_{\mathrm{eff}}=\left|\sum_{i=1}^{9} U_{e i}^{2} \frac{\bar{p}^{2}}{\bar{p}^{2}+m_{i}^{2}} m_{i}\right| \simeq\left|\left(\sum_{i=1}^{3} U_{e i}^{2} m_{i}\right)+m_{\mathrm{eff}}^{N}\right|,
$$

where $\bar{p}^{2} \sim(200 \mathrm{MeV})^{2}$ is the typical virtual momentum of the neutrino. The first summation term corresponds to contributions from the active neutrinos, and $m_{\mathrm{eff}}^{N}$ stands for the heavy neutrino contributions. In the inverse seesaw model, $m_{\mathrm{eff}}^{N}$ can be obtained by $[54,55]$

$$
\begin{aligned}
m_{\mathrm{eff}}^{N} \simeq & \sum_{i=1}^{3}\left[-U_{e(2 i+2)}^{2} \frac{\bar{p}^{2}}{\bar{p}^{2}+m_{i-}^{2}}\left|m_{i-}\right|+U_{e(2 i+3)}^{2} \frac{\bar{p}^{2}}{\bar{p}^{2}+m_{i+}^{2}}\left|m_{i+}\right|\right] \\
= & -\sum_{i=1}^{3}\left[\left(U_{e(2 i+2)}^{2}+U_{e(2 i+3)}^{2}\right) \frac{\left[\left(M_{i}^{2}-\mu_{i}^{2}\right)-\bar{p}^{2}\right] \bar{p}^{2}}{\left[\left(M_{i}^{2}-\mu_{i}^{2}\right)+\bar{p}^{2}\right]^{2}+4 \bar{p}^{2} \mu_{i}^{2}} \mu_{i}\right. \\
& \left.+\left(U_{e(2 i+2)}^{2}-U_{e(2 i+3)}^{2}\right) \frac{\left[\left(M_{i}^{2}-\mu_{i}^{2}\right)+\bar{p}^{2}\right] \bar{p}^{2}}{\left[\left(M_{i}^{2}-\mu_{i}^{2}\right)+\bar{p}^{2}\right]^{2}+4 \bar{p}^{2} \mu_{i}^{2}} M_{i}\right]
\end{aligned}
$$

In the limit of $\mu_{i} \rightarrow 0$ (no lepton number asymmetry), the heavy neutrino contributions exactly vanish due to $U_{e 4}^{2}=U_{e 5}^{2}, U_{e 6}^{2}=U_{e 7}^{2}$ and $U_{e 8}^{2}=U_{e 9}^{2}$. Notice that we can usually take $\left(M_{i}^{2}-\mu_{i}^{2}\right)^{1 / 2} \simeq M_{i}$, but this approximation becomes invalid if one considers leptogenesis thorough neutrino oscillations to explain the BAU, in which $0.1 \lesssim 2 \mu_{i} / M_{i} \lesssim 1$ [51].

In addition, the unitary matrix $U$ has the following approximate relations:

$$
U_{\alpha(2 i+2)}^{2}+U_{\alpha(2 i+3)}^{2} \simeq \frac{\left(m_{D}^{*}\right)_{i \alpha}^{2}}{M_{i}^{2}} \quad \text { and } \quad U_{\alpha(2 i+2)}^{2}-U_{\alpha(2 i+3)}^{2} \simeq \frac{\left(m_{D}^{*}\right)_{i \alpha}^{2}}{M_{i}^{2}} \frac{2 \mu_{i}}{M_{i}}
$$

where $\alpha=e, \mu$ and $\tau$. Then, we find the analytic form of $m_{\mathrm{eff}}^{N}$ as

$$
m_{\mathrm{eff}}^{N} \simeq-\sum_{i=1}^{3} \frac{\bar{p}^{2}}{\left(M_{i}^{2}-\mu_{i}^{2}\right)+\bar{p}^{2}}\left[2+\frac{\left(M_{i}^{2}-\mu_{i}^{2}\right)-\bar{p}^{2}}{\left(M_{i}^{2}-\mu_{i}^{2}\right)+\bar{p}^{2}}\right]\left(\tilde{m}_{\nu}^{*}\right)_{i e}
$$



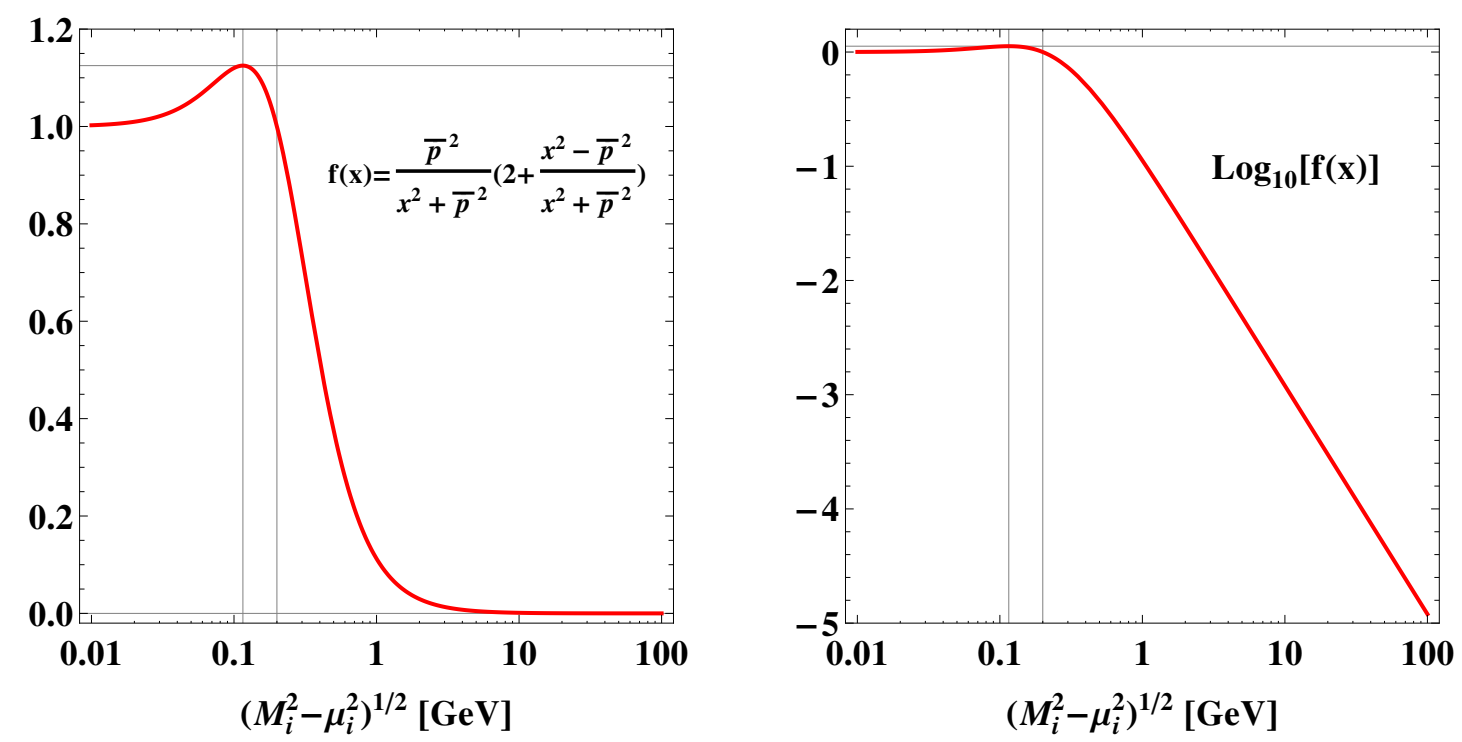

Figure 1. The suppression factor in eq. (3.4). The vertical lines show $\sqrt{\bar{p}^{2}}=200 \mathrm{MeV}$ and $\sqrt{\bar{p}^{2} / 3}$. The horizontal line show $9 / 8$ as the maximal value. The right panel is shown with a logarithmic scale.

with

$$
\left(\tilde{m}_{\nu}\right)_{i e}=\frac{\left(m_{D}\right)_{i e}^{2} \mu_{i}}{M_{i}^{2}}
$$

The coefficient factor in eq. (3.4) is strongly suppressed for a large $\left(M_{i}^{2}-\mu_{i}^{2}\right)^{1 / 2}$. Figure 1 shows it as a function of $\left(M_{i}^{2}-\mu_{i}^{2}\right)^{1 / 2}$. From figure 1, we can see that sizable $m_{\text {eff }}^{N}$ is likely to be obtained by $\left(M_{i}^{2}-\mu_{i}^{2}\right)^{1 / 2} \lesssim 1 \mathrm{GeV}$. Since $\left(\tilde{m}_{\nu}\right)_{i e}$ appears in the active neutrino mass matrix, i.e. $\left(m_{\nu}\right)_{e e} \simeq \sum_{i=1}^{3}\left(\tilde{m}_{\nu}\right)_{i e}$ [see eq. (2.8)], $\left(\tilde{m}_{\nu}\right)_{i e}$ is typically $\mathcal{O}\left(m_{\nu}\right)$ without any fine-tuning. However, it can be much larger than $\mathcal{O}\left(m_{\nu}\right)$ with a sufficient fine-tuning, so that $m_{\mathrm{eff}}^{N}$ can be much larger than the naive estimation. We will show numerical results in section 5 .

The existence of heavy neutrinos also induce non-standard interactions in the leptonic sector, which correspond to non-unitarity of the PMNS matrix. The deviation from unitarity can be estimated by

$$
\epsilon_{\alpha \beta} \equiv\left|\sum_{i=4}^{7} U_{\alpha i} U_{\beta i}^{*}\right|=\left|\delta_{\alpha \beta}-\left(N N^{\dagger}\right)_{\alpha \beta}\right|,
$$

where $N$ is the $3 \times 3$ non-unitary matrix describing the mixing between the light neutrino mass eigenstates and the $\mathrm{SU}(2)_{L}$ gauge eigenstates, that is, the PMNS matrix. The values of $\epsilon_{\alpha \beta}$ are severely constrained by the combined data from neutrino oscillation data, leptonflavor-violating decays of charged leptons, non-universality of weak interaction, CKM uni- 
tarity bounds, and EW precision data [56]:

$$
\left|\epsilon_{\alpha \beta}\right| \leq\left(\begin{array}{lll}
2.5 \times 10^{-3} & 2.4 \times 10^{-5} & 2.7 \times 10^{-3} \\
2.4 \times 10^{-5} & 4.0 \times 10^{-4} & 1.2 \times 10^{-3} \\
2.7 \times 10^{-3} & 1.2 \times 10^{-3} & 5.6 \times 10^{-3}
\end{array}\right)
$$

The constraint on $\epsilon_{e \mu}\left(=\epsilon_{\mu e}\right)$, which comes from a constraint on the lepton-flavor-violating muon decay $\mu \rightarrow e \gamma$, is much stronger than the others. Without assuming accidental cancellation or special textures for the neutrino Yukawa coupling matrix (that is, all components of Yukawa coupling matrix have the same order values), once model parameters are set to satisfy $\epsilon_{e \mu} \leq 2.4 \times 10^{-5}$, the other constraints can be simultaneously satisfied.

\section{Higgs mass correction}

The heavy neutrinos may lead a sizable Higgs mass correction. In our notation, the Higgs potential is given by $V=\lambda\left(\Phi^{\dagger} \Phi\right)^{2}+m_{H}^{2} \Phi^{\dagger} \Phi$, and then, the Higgs mass is obtained by $M_{h}^{2}=$ $-2 m_{H}^{2}=(125 \mathrm{GeV})^{2}$. Then, the heavy neutrinos contribute the Higgs mass parameter as

$$
\begin{aligned}
\delta m_{H}^{2} & =-\frac{2}{16 \pi^{2}} \operatorname{Tr}\left[Y_{\nu}^{\dagger} M_{N}^{T} M_{N} Y_{\nu}\right] \ln \left(\frac{M_{\mathrm{Pl}}^{2}}{M_{N}^{2}}\right) \\
& =-\frac{4}{16 \pi^{2} v^{2}} \operatorname{Tr}\left[D_{M}^{2} D_{X} R D_{m} R^{\dagger}\right] \ln \left(\frac{M_{\mathrm{Pl}}^{2}}{M_{N}^{2}}\right),
\end{aligned}
$$

where we have used Casas-Ibarra parametrization (2.10), and assumed both $M_{N}$ and $\mu_{S}$ are diagonal. We have taken cutoff scale as the reduced Planck scale $M_{\mathrm{Pl}}=2.44 \times 10^{18} \mathrm{GeV}$. In the last expression of eq. (4.1), $D_{X} \rightarrow D_{M}$ corresponds to the Higgs mass correction in the type-I seesaw model. Thus, in the inverse seesaw model, the Higgs mass correction can be enhanced by a factor $M_{N} / \mu_{S}$ compared to the type-I seesaw model.

Since we have assumed that both $M_{N}$ and $\mu_{S}$ are diagonal, the trace part in eq. (4.1) can be simply shown by

$$
\operatorname{Tr}\left[D_{M}^{2} D_{X} R D_{m} R^{\dagger}\right]=\left(\frac{M_{1}^{4}}{\mu_{1}}, \frac{M_{2}^{4}}{\mu_{2}}, \frac{M_{3}^{4}}{\mu_{3}}\right)\left(\begin{array}{c}
\left|R_{11}\right|^{2}\left|R_{12}\right|^{2}\left|R_{13}\right|^{2} \\
\left|R_{21}\right|^{2}\left|R_{22}\right|^{2}\left|R_{23}\right|^{2} \\
\left|R_{31}\right|^{2}\left|R_{32}\right|^{2}\left|R_{33}\right|^{2}
\end{array}\right)\left(\begin{array}{c}
m_{1} \\
m_{2} \\
m_{3}
\end{array}\right) .
$$

In addition, when both $M_{N}$ and $\mu_{S}$ are degenerate, i.e., $M_{N}=M_{d} \mathbf{1}$ and $\mu_{S}=\mu_{d} \mathbf{1}$, this trace can be rewritten by

$$
\operatorname{Tr}\left[D_{M}^{2} D_{X} R D_{m} R^{\dagger}\right]=\frac{M_{d}^{4}}{\mu_{d}}\left(R_{1} m_{1}+R_{2} m_{2}+R_{3} m_{3}\right),
$$

with $R_{i} \equiv \sum_{j=1}^{3}\left|R_{j i}\right|^{2}$, which is obtained by

$$
\begin{aligned}
& R_{1}=\left|c_{12}\right|^{2}\left|c_{13}\right|^{2}+\left(\left|s_{12}\right|^{2}+\left|c_{12}\right|^{2}\left|s_{13}\right|^{2}\right) \cosh \left(2 \operatorname{Im}\left[\omega_{23}\right]\right)+2 \operatorname{Im}\left[s_{12} c_{12}^{*} s_{13}^{*}\right] \sinh \left(2 \operatorname{Im}\left[\omega_{23}\right]\right), \\
& R_{2}=\left|s_{12}\right|^{2}\left|c_{13}\right|^{2}+\left(\left|c_{12}\right|^{2}+\left|s_{12}\right|^{2}\left|s_{13}\right|^{2}\right) \cosh \left(2 \operatorname{Im}\left[\omega_{23}\right]\right)+2 \operatorname{Im}\left[s_{12} c_{12}^{*} s_{13}\right] \sinh \left(2 \operatorname{Im}\left[\omega_{23}\right]\right), \\
& R_{3}=\left|s_{13}\right|^{2}+\left|c_{13}\right|^{2} \cosh \left(2 \operatorname{Im}\left[\omega_{23}\right]\right) .
\end{aligned}
$$


In this expression, we can see $R_{i} \geq 1$. This fact is easily understood in two flavor case, in which the number of right-handed neutrinos and sterile neutrinos are two. In the two flavor case, the lightest neutrino is massless, and $R$ is expressed by only one complex angle. The normal hierarchy $(\mathrm{NH})$ corresponds to $m_{1}=0$ and $\omega_{12}=\omega_{13}=0$, which lead $R_{2}=R_{3}=\cosh \left(2 \operatorname{Im}\left[\omega_{23}\right]\right) \geq 1$. In the same way, the inverted hierarchy (IH) corresponds to $m_{3}=0$ and $\omega_{13}=\omega_{23}=0$, which lead $R_{1}=R_{2}=\left|c_{12}\right|^{2}+\left|s_{12}\right|^{2}=\cosh \left(2 \operatorname{Im}\left[\omega_{12}\right]\right) \geq 1$.

As a result, the Higgs mass correction has the minimal value:

$$
\left|\delta m_{H}^{2}\right| \geq \frac{4 M_{d}^{4}}{16 \pi^{2} v^{2} \mu_{d}}\left(m_{1}+m_{2}+m_{3}\right) \ln \left(\frac{M_{\mathrm{Pl}}^{2}}{M_{d}^{2}}\right)
$$

where the equals sign holds with, e.g., $R=1$. For the non-degenerate case, $M_{d}$ is replaced by the heaviest neutrino mass. Figure 2 shows the Higgs mass correction as a function of $\mu_{d}$, which corresponds to the minimal value of eq. (4.5). For reference values, we have considered the NH case, and taken $m_{1}=0, m_{2}=\sqrt{7.49 \times 10^{-5}} \mathrm{eV}$ and $m_{3}=\sqrt{2.484 \times 10^{-3}} \mathrm{eV}$ [57]. The red, pink, green, cyan and blue lines show $\left|\delta m_{H}^{2}\right|$ for $M_{d}=1,10,10^{2}, \cdots$, and $10^{5} \mathrm{GeV}$, respectively. Using the seesaw relation (2.6), typical values of neutrino Yukawa coupling can be determined by a function of $\mu_{d}$ with a fixed $M_{d}$, which are shown in the gray-dashed lines. From figure 2, we can see that the Higgs mass correction becomes larger as $\mu_{d}$ becomes smaller. When $\mu_{d}$ is smaller than $0.1 \mathrm{eV}$, the inverse seesaw mechanism does not work successfully as shown in eq. (2.7). For $\mu_{d}>0.1 \mathrm{eV}$, the heavy neutrino contributions to the Higgs mass become dominant for $M_{d} \gtrsim 160 \mathrm{GeV}$. This fact is much different from the type-I seesaw model, in which $\left|\delta m_{H}^{2}\right|<M_{h}^{2} / 2$ for $M_{d}<10^{6} \mathrm{GeV}$. Therefore, from the naturalness point of view, inverse seesaw models have to introduce heavy neutrinos more carefully than the type-I seesaw model.

\section{$5 \quad$ Numerical analysis}

In this section, we show numerical results of the effective neutrino mass (3.1) and the Higgs mass correction (4.1). We focus on the case where $M_{N}$ and $\mu_{N}=\mu_{S}$ are real diagonal matrices and normally hierarchical $M_{N}$, i.e., $M_{1}<M_{2}<M_{3}$. Actually, even if they are not normally hierarchical, we have found similar conclusions for other hierarchies. In our numerical calculations, we take the input parameters as

$$
\begin{aligned}
m_{1(3)} & =\left[10^{-4} \mathrm{eV}, 0.07(0.065) \mathrm{eV}\right] \quad \text { for } \mathrm{NH}(\mathrm{IH}), \\
M_{i} & =[10 \mathrm{MeV}, 100 \mathrm{TeV}] \\
\mu_{i} & =[1 \mathrm{eV}, 1 \mathrm{MeV}] \\
\omega_{i j} & =[0, \pi] \times e^{i[0,2 \pi]}
\end{aligned}
$$

and neutrino oscillation parameters satisfy the current experimental constraints shown in table 1. The upper bound of the lightest active neutrino mass is given by the cosmological bound $\sum m_{i}<0.23 \mathrm{eV}$ [58]. In addition, the mixing matrix $U$ satisfies the constraints of the non-unitarity (3.7). Note that, however, there exist severe constraints in a low $M_{i}$ region $\left(M_{i} \lesssim 2 \mathrm{GeV}\right)$, which are obtained by $\pi$ and $K$ peak searches, $\pi, K, D, Z$ decay 


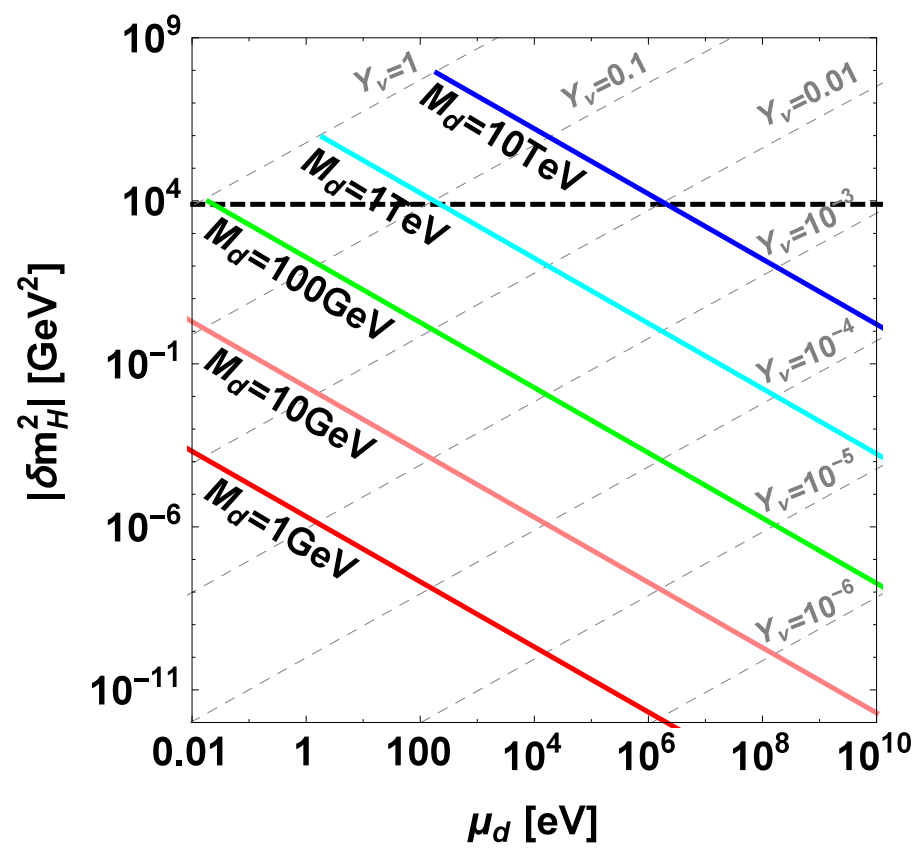

Figure 2. Higgs mass correction as a function of $\mu_{d}$, which are shown by the red, pink, green, cyan and blue lines for $M_{d}=1,10,10^{2}, \cdots$, and $10^{5} \mathrm{GeV}$, respectively. The blackdashed line corresponds to $\left|\delta m_{H}^{2}\right|=M_{h}^{2} / 2$ with $M_{h}=125 \mathrm{GeV}$. Typical values of neutrino Yukawa coupling can be estimated by eq. (2.6), and they are shown by the gray-dashed lines for $Y_{\nu}=1,10^{-1}, 10^{-2}, \cdots$, and $10^{-6}$ from the upper left to the lower right.

\begin{tabular}{|c|ccccc|}
\hline & $\sin ^{2} \theta_{12}$ & $\sin ^{2} \theta_{23}$ & $\sin ^{2} \theta_{13}$ & $\frac{\Delta m_{21}^{2}}{10^{-5} \mathrm{eV}^{2}}$ & $\frac{\Delta m_{3 \ell}^{2}}{10^{-3} \mathrm{eV}^{2}}$ \\
\hline $\mathrm{NH}$ & $0.273 \rightarrow 0.349$ & $0.390 \rightarrow 0.639$ & $0.0187 \rightarrow 0.0250$ & $7.02 \rightarrow 8.08$ & $2.351 \rightarrow 2.618$ \\
$\mathrm{IH}$ & $0.273 \rightarrow 0.349$ & $0.400 \rightarrow 0.637$ & $0.0190 \rightarrow 0.0251$ & $7.02 \rightarrow 8.08$ & $2.341 \rightarrow 2.595$ \\
\hline
\end{tabular}

Table 1. Global fit values of neutrino oscillation parameters in $3 \sigma$ CL range [57]. The mass squared differences are defined by $\Delta m_{21}^{2}=m_{2}^{2}-m_{1}^{2}$ and $\Delta m_{3 \ell}^{2}=\left|m_{3}^{2}-m_{\ell}^{2}\right|$ with $\ell=1$ and 2 for the NH and the IH cases, respectively. There are no constraints for all CP phases in $3 \sigma$ CL range.

searches, and LHC collider searches. They are summarized in ref. [23], and we also apply their constraints in our analysis.

For the neutrinoless double beta decay, the lightest heavy neutrino mass $M_{1}$ is sensitive. Figure 3 shows $M_{1}$ dependence of $\left|m_{\mathrm{eff}}^{N}\right|$ with the blue dots, while the red dots show the active neutrino contribution $\left|m_{\text {eff }}^{\nu}\right|$, which does not depend on heavy neutrinos. The cyan and pink dots show the excluded points due to the constraints in ref. [23] for $\left|m_{\mathrm{eff}}^{N}\right|$ and $\left|m_{\text {eff }}^{\nu}\right|$, respectively. The behavior of $\left|m_{\text {eff }}^{N}\right|$ is the same as figure 1, which is expected from our analytical result (3.4). The heavy neutrino contribution can be much larger than the active neutrino contribution in the range of $M_{1} \lesssim 1 \mathrm{GeV}$.

Figure 4 shows $M_{1}$ dependence of $m_{\mathrm{eff}}$ (blue dots). The cyan dots show the excluded points due to the constraints in ref. [23]. The gray band and the black-dashed line corresponds to the current upper bound $m_{\text {eff }}<(61-165) \mathrm{meV}$ obtained by the KamLAND-Zen 

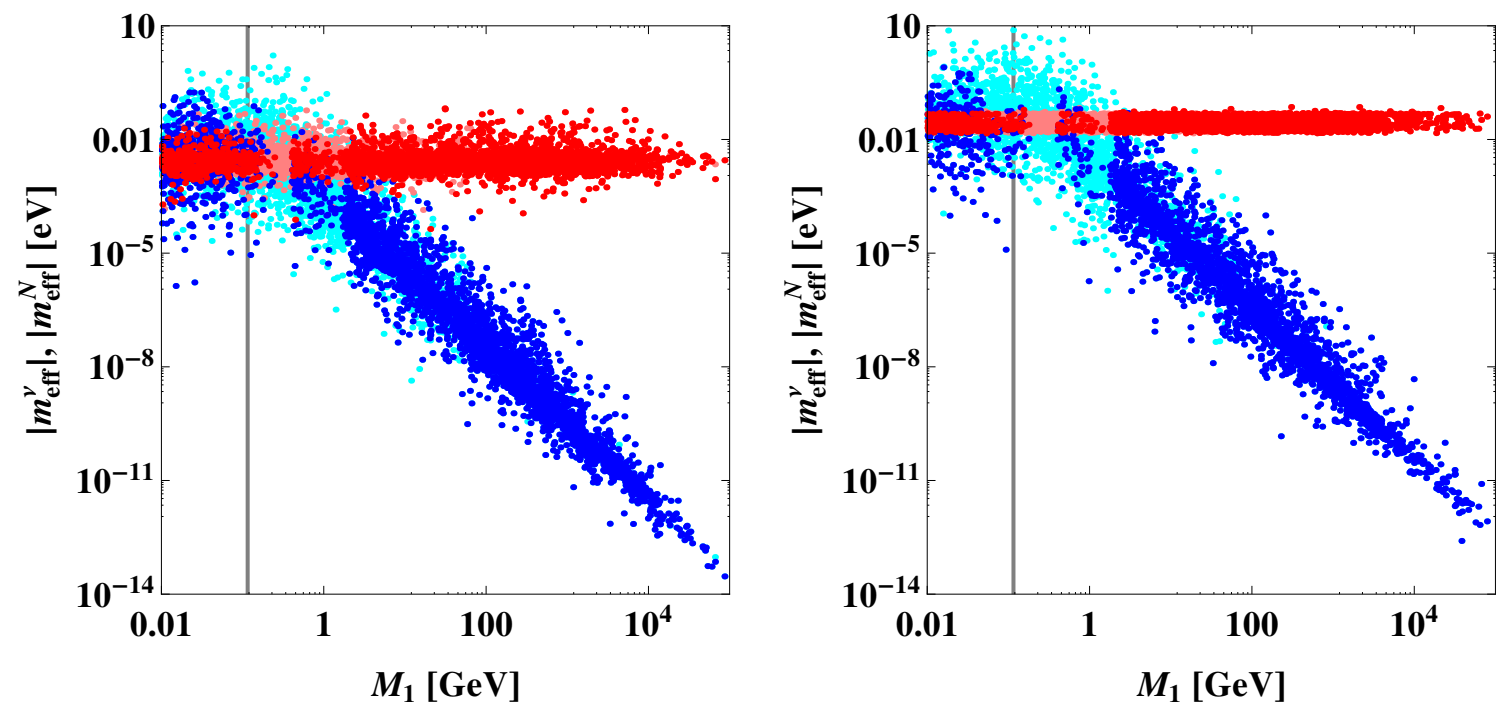

Figure 3. Heavy neutrino mass dependence of $\left|m_{\text {eff }}^{N}\right|$ (blue dots). The red dots show $\left|m_{\text {eff }}^{\nu}\right|$, which does not depend on heavy neutrinos. The cyan and pink dots show the excluded points due to the constraints in ref. [23] for $\left|m_{\text {eff }}^{N}\right|$ and $\left|m_{\text {eff }}^{\nu}\right|$, respectively. The vertical line shows $\sqrt{\bar{p}^{2} / 3}$ with $\sqrt{\bar{p}^{2}}=200 \mathrm{MeV}$. The left and right panels correspond to the NH and the IH cases, respectively.
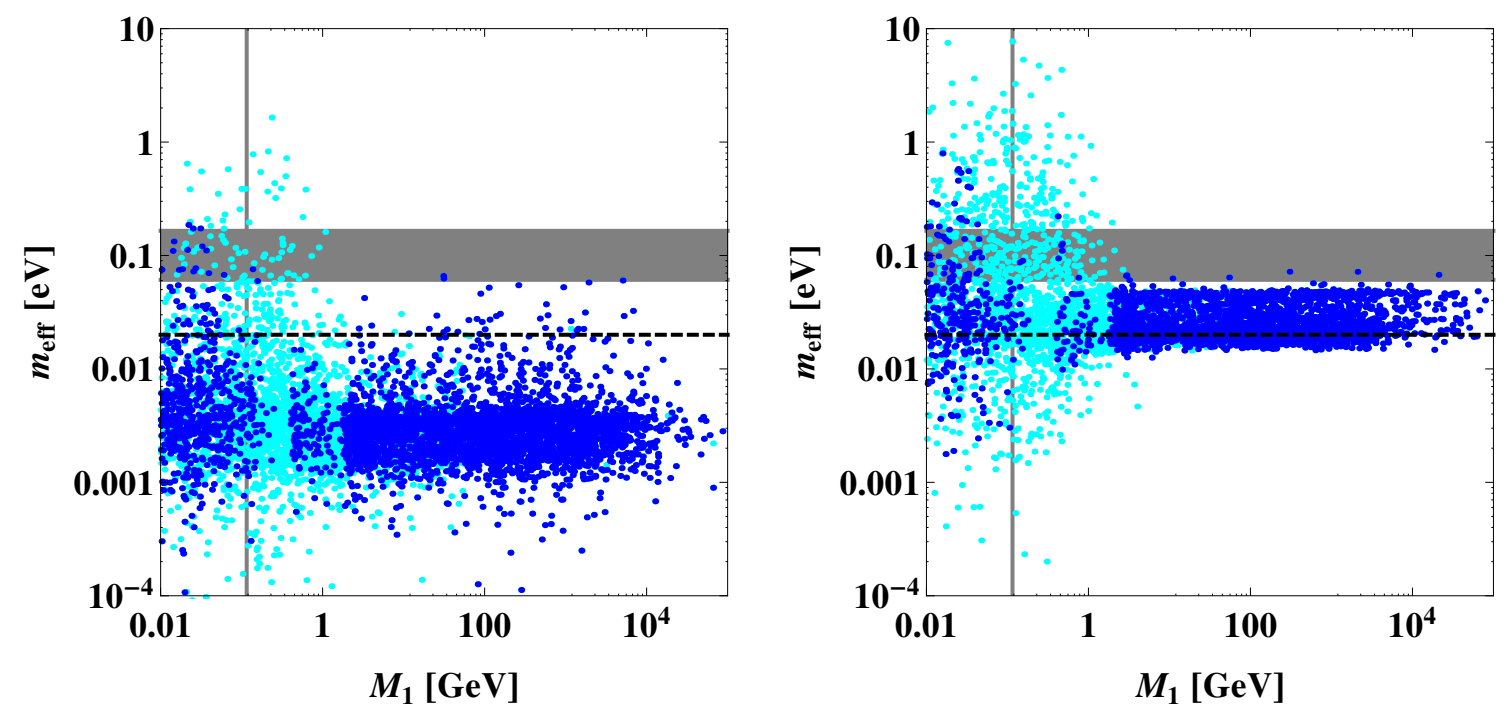

Figure 4. Heavy neutrino mass dependence of $m_{\text {eff }}$ (blue dots). The cyan dots show the excluded points due to the constraints in ref. [23]. The gray band and the black-dashed line show the current upper bound $m_{\mathrm{eff}}<(61-165) \mathrm{meV}$ and the future sensitivity $m_{\mathrm{eff}}<0.02 \mathrm{eV}$, respectively. The vertical line shows $\sqrt{\bar{p}^{2} / 3}$ with $\sqrt{\bar{p}^{2}}=200 \mathrm{MeV}$. The left and right panels correspond to the NH and the IH cases, respectively. 

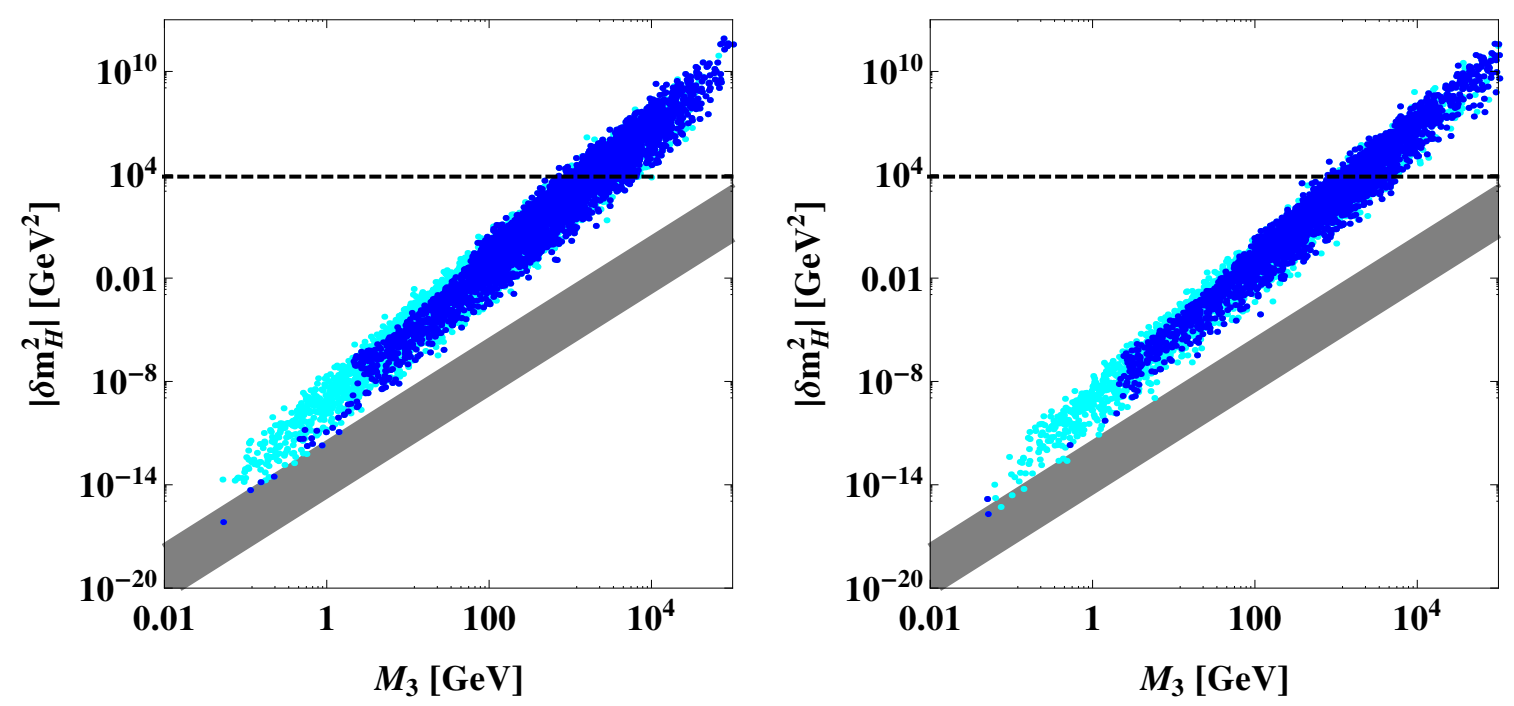

Figure 5. Heavy neutrino mass dependence of the Higgs mass correction (blue dots). The cyan dots show the excluded points due to the constraints in ref. [23]. The gray band and the blackdashed line show the type-I seesaw case, and $\left|\delta m_{H}^{2}\right|=M_{h}^{2} / 2$ with $M_{h}=125 \mathrm{GeV}$, respectively. The left and right panels correspond to the $\mathrm{NH}$ and the $\mathrm{IH}$ cases, respectively.

experiment [18] and their future sensitivity $m_{\mathrm{eff}}<0.02 \mathrm{eV}$, respectively. For $M_{1}>1 \mathrm{GeV}$, the heavy neutrino contribution is strongly suppressed, and thus, there are almost no points above the current upper bound. Note that there exists $m_{\text {eff }}<\left|m_{\text {eff }}^{\nu}\right|$ region, since $m_{\text {eff }}^{\nu}$ and $m_{\mathrm{eff}}^{N}$ can be canceled each other. Thus, the IH case is not completely excluded.

For the Higgs mass correction, the heaviest heavy neutrino mass $M_{3}$ is sensitive. Figure 5 shows $M_{3}$ dependence of $\left|\delta m_{H}^{2}\right|$ (blue dots). The cyan dots show the excluded points due to the constraints in ref. [23]. The gray band shows the type-I seesaw case, and the black-dashed line corresponds to $\left|\delta m_{H}^{2}\right|=M_{h}^{2} / 2$ with $M_{h}=125 \mathrm{GeV}$. The minimal value of Higgs mass correction can be predicted by eq. (4.5). For the maximal value of $\operatorname{Im}\left[\omega_{i j}\right]=\omega_{\max }$ (in our numerical analysis $\omega_{\max }=\pi$ ), the maximal value of Higgs mass correction is approximately given by the minimal value times $\cosh \left(2 \omega_{\max }\right)$. There is no difference between the $\mathrm{NH}$ and the IH cases. Note that $M_{i} \gtrsim 10^{5} \mathrm{GeV}$, which equivalently corresponds to $Y_{\nu} \gtrsim 1$, is excluded by the constraint from lepton flavor violations. ${ }^{4}$ If we allow $\mu_{i}$ to take a larger value than $1 \mathrm{MeV}, Y_{\nu}$ can be smaller than before, and then, there exist allowed regions for $M_{i} \gtrsim 10^{5} \mathrm{GeV}$. However, since such a large $\mu_{i}$ means a large lepton number violation, it conflicts the naturalness. Thus, we have imposed $\mu_{i} \leq 1 \mathrm{MeV}$.

As we expected in section 4, the Higgs mass correction can be larger than the Higgs mass for $M_{3} \gtrsim 1 \mathrm{TeV}$, while for $M_{3} \gtrsim 10^{6} \mathrm{GeV}$ in the type-I seesaw model. This difference corresponds to the difference of size of neutrino Yukawa coupling, that is, in the inverse seesaw model $Y_{\nu}$ is much lager compared with the type-I seesaw case. The large $Y_{\nu}$ causes

\footnotetext{
${ }^{4}$ The electroweak vacuum becomes instable before the Planck scale for $\operatorname{Tr}\left[Y_{\nu}^{\dagger} Y_{\nu}\right] \gtrsim 0.4$ [59], and we have checked that our numerical results shown in figures 3-6 can avoid the vacuum instability except for a few points, at which $M_{3} \simeq 10^{5} \mathrm{GeV}$.
} 

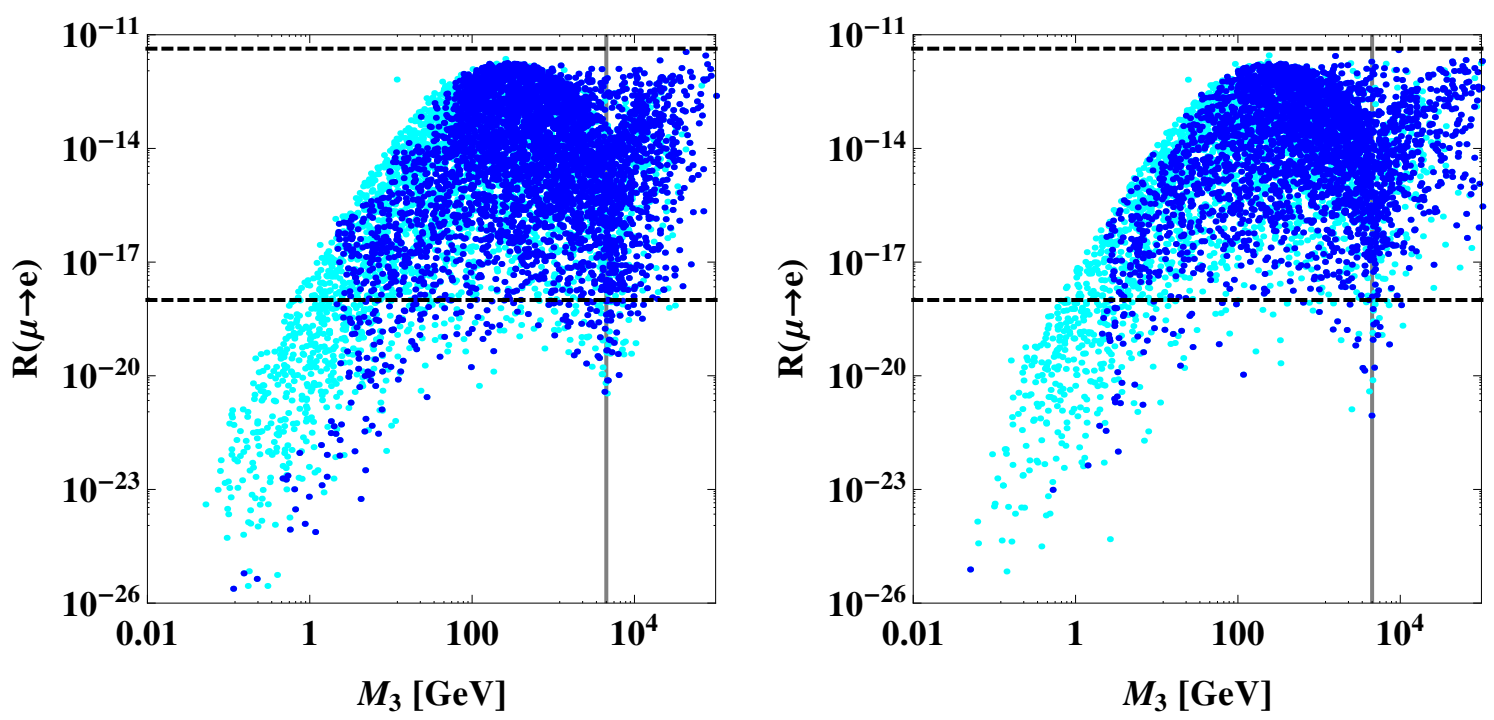

Figure 6. The rate of $\mu \rightarrow e$ conversion in Titanium (blue dots). The cyan dots show the excluded points due to the constraints in ref. [23]. The black-dashed lines correspond to the current upper bound $R_{\mu \rightarrow e}<4.3 \times 10^{-12}$ and the future sensitivity of PRISM experiment $R_{\mu \rightarrow e}<10^{-18}$. The vertical line shows $M_{3}=4.5 \mathrm{TeV}$, at which the contribution of the $\mu \rightarrow e$ conversion vanishes. The left and right panels correspond to the $\mathrm{NH}$ and the $\mathrm{IH}$ cases, respectively.

a large mixing between left-handed neutrinos and gauge-singlet neutrinos. However, such a large mixing can be severely constrained by future experiments of the lepton flavor violation. In particular, a future experiment of $\mu \rightarrow e$ conversion at PRISM can give the strongest constraint.

Figure 6 shows the rate of $\mu \rightarrow e$ conversion in Titanium (blue dots), which has been calculated as in appendix A. ${ }^{5}$ The cyan dots show the excluded points due to the constraints in ref. [23]. The black-dashed line corresponds to the future sensitivity of PRISM experiment $R_{\mu \rightarrow e}<10^{-18}$ [30], while the current upper bound is $R_{\mu \rightarrow e}<4.3 \times$ $10^{-12}[61]$. For Titanium, the $\mu \rightarrow e$ conversion rate vanishes at $M_{i} \simeq 4.5 \mathrm{TeV}$. Since the vanishing point is the different for the various nuclei, the experiment using Titanium can be complemented by experiments using other nuclei. In addition, the low mass region $M_{i} \lesssim$ $140 \mathrm{MeV}$ may be excluded by constraints coming from the big bang nucleosynthesis [62]. Therefore, we can expect the inverse seesaw model is highly testable, i.e., future experiments of neutrinoless double beta decay and $\mu \rightarrow e$ conversion can search the low mass region $M_{i} \lesssim 1 \mathrm{GeV}$ and the high mass region $M_{i} \gtrsim 1 \mathrm{GeV}$, respectively. ${ }^{6}$

Finally, we mention our assumption " $M_{N}$ and $\mu_{N}=\mu_{S}$ are diagonal". If this assumption were relaxed, the matrix $V$ is not the identity matrix, which can change the

\footnotetext{
${ }^{5}$ Impact of heavy neutrinos on charged-lepton flavor violation in the inverse seesaw model has been also addressed in ref. [60].

${ }^{6}$ If we allow $\mu_{i}$ to take a larger value than $1 \mathrm{MeV}$, there is more allowed region which has a smaller $Y_{\nu}$. Actually, the BAU can be explained only in such a parameter space [51], although it is not preferred from the naturalness point of view.
} 
configuration of the Yukawa coupling matrix $Y_{\nu}$ (see eq. (2.10)). However, this new degree of freedom does not change the order of magnitude of the matrix, and the matrix $R$ can also change the configuration of $Y_{\nu}$. Thus, we can expect that the effect of $V$ does not change allowed parameter space of $Y_{\nu}$ (at least significantly). Actually, in the numerical calculations, the matrix $U$ is rather effective than $Y_{\nu}$, and we have checked that all scatter plots of $\left(M_{i},\left|U_{i j}\right|^{2}\right)$ are uniformly distributed, which means our assumption $\left(M_{N}\right.$ and $\mu_{N}=\mu_{S}$ are diagonal) does not restrict the results. After all, we can expect that, even if the assumption were relaxed, our statement does not change.

\section{Summary}

We focus on the $(3,3)$ inverse seesaw model, in which the number of both right-handed neutrinos and sterile neutrinos are three. We have investigated heavy neutrino contributions for the neutrinoless double beta decay. Its rate is proportional to the effective neutrino mass, and it is useful to estimate contributions from the heavy neutrinos. We have found an analytic form of the heavy neutrino contribution to the effective neutrino mass. It is strongly suppressed for a large heavy-neutrino mass $\gtrsim 1 \mathrm{GeV}$, while, in $\sim 0.1 \mathrm{GeV}$ region, it can be enhanced by ten times or more than the active neutrino contribution alone. We have also investigated the Higgs mass correction coming from the heavy neutrinos, and found the minimal value of Higgs mass correction is determined for a given heavy neutrino mass, which is usually larger than the type-I seesaw case. Then, we have shown numerical results of heavy neutrino contributions to the effective neutrino mass and the Higgs mass correction. As a result, we have found that almost all parameter space of the inverse seesaw model can be complementarily searched: the low mass region $M_{i} \lesssim 1 \mathrm{GeV}$ and the high mass region $M_{i} \gtrsim 1 \mathrm{GeV}$ can be searched by future experiments of neutrinoless double beta decay and $\mu \rightarrow e$ conversion, respectively.

\section{Acknowledgments}

The authors thank C. Weiland for the important comments about footnotes 3 and 4 . This work is partially supported by Scientific Grants by the Ministry of Education, Culture, Sports, Science and Technology of Japan (Nos. 24540272, 26247038, 15H01037, 16H00871, and 16H02189). The work of Y.Y. is supported by Research Fellowships of the Japan Society for the Promotion of Science for Young Scientists (Grants No. 26 2428).

\section{A $\quad \mu \rightarrow e$ conversion rate}

Due to the existence of the heavy neutrinos, violation of charged lepton number arises at the one loop level. $\mu \rightarrow e$ conversion is induced by a series of gauge boson mediated diagrams. Its rate is calculated by [63] (see also ref. [64] for the inverse seesaw model with or without supersymmetry)

$$
R_{\mu \rightarrow e} \simeq \frac{G_{\mathrm{F}}^{2} \alpha_{W}^{2} \alpha^{3} m_{\mu}^{5}}{8 \pi^{4} \Gamma_{\text {capt }}} \frac{Z_{\mathrm{eff}}^{4}}{Z} F_{p}^{2}\left|\sum_{i=1}^{9}\left[(A+Z) F_{u}\left(x_{i}\right)+(2 A-Z) F_{d}\left(x_{i}\right)\right] U_{e i} U_{\mu i}^{*}\right|^{2} .
$$


where $\alpha_{W}=g_{2}^{2} /(4 \pi), \alpha=e^{2} /(4 \pi), s_{W}=\sin \theta_{W}$ is the Weinberg angle, $G_{\mathrm{F}}$ is the Fermi constant, and $m_{\mu}$ is the muon mass. The other constant parameters depend on a nuclei information which is used in experiments. $A$ is the mass number, $Z$ ( $\left.Z_{\text {eff }}\right)$ is the (effective) atomic number, $F_{p}$ is a nuclear form factor, and $\Gamma_{\text {capt }}$ is the capture rate. These values are given in table 2 [63].

$F_{u}\left(x_{i}\right)$ and $F_{d}\left(x_{i}\right)$ are functions of $x_{i} \equiv m_{i}^{2} / M_{W}^{2}$ given as

$$
\begin{aligned}
\tilde{F}_{u}(x)= & \frac{2}{3} s_{W}^{2}\left[F_{\gamma}(x)-F_{Z}(x)-2 G_{Z}(0, x)\right] \\
& +\frac{1}{4}\left[F_{Z}(x)+2 G_{Z}(0, x)+F_{\mathrm{Box}}(0, x)-F_{\mathrm{Box}}(0,0)\right], \\
\tilde{F}_{d}(x)= & -\frac{1}{3} s_{W}^{2}\left[F_{\gamma}(x)-F_{Z}(x)-2 G_{Z}(0, x)\right] \\
& -\frac{1}{4}\left[F_{Z}(x)+2 G_{Z}(0, x)-F_{\mathrm{XBox}}(0, x)+F_{\mathrm{XBox}}(0,0)\right], \\
F_{u}(x)= & \tilde{F}_{u}(x)+\frac{2}{3} s_{W}^{2} G_{\gamma}(x), \\
F_{d}(x)= & \tilde{F}_{d}(x)-\frac{1}{3} s_{W}^{2} G_{\gamma}(x) .
\end{aligned}
$$

The loop functions are

$$
\begin{aligned}
F_{\gamma}(x)= & \frac{x\left(7 x^{2}-x-12\right)}{12(1-x)^{3}}-\frac{x^{2}\left(x^{2}-10 x+12\right)}{6(1-x)^{4}} \ln x, \\
G_{\gamma}(x)= & -\frac{x\left(2 x^{2}+5 x-1\right)}{4(1-x)^{3}}-\frac{3 x^{3}}{2(1-x)^{4}} \ln x, \\
F_{Z}(x)= & -\frac{5 x}{2(1-x)}-\frac{5 x^{2}}{2(1-x)^{2}} \ln x, \\
G_{Z}(x, y)= & -\frac{1}{2(x-y)}\left[\frac{x^{2}(1-y)}{1-x} \ln x-\frac{y^{2}(1-x)}{1-y} \ln y\right], \\
F_{\text {Box }}(x, y)= & \frac{1}{x-y}\left\{\left(4+\frac{x y}{4}\right)\left[\frac{1}{1-x}+\frac{x^{2}}{(1-x)^{2}} \ln x-\frac{1}{1-y}-\frac{y^{2}}{(1-y)^{2}} \ln y\right]\right. \\
& \left.-2 x y\left[\frac{1}{1-x}+\frac{x}{(1-x)^{2}} \ln x-\frac{1}{1-y}-\frac{y}{(1-y)^{2}} \ln y\right]\right\}, \\
F_{\mathrm{XBox}}(x, y)= & \frac{-1}{x-y}\left\{\left(1+\frac{x y}{4}\right)\left[\frac{1}{1-x}+\frac{x^{2}}{(1-x)^{2}} \ln x-\frac{1}{1-y}-\frac{y^{2}}{(1-y)^{2}} \ln y\right]\right. \\
& \left.-2 x y\left[\frac{1}{1-x}+\frac{x}{(1-x)^{2}} \ln x-\frac{1}{1-y}-\frac{y}{(1-y)^{2}} \ln y\right]\right\},
\end{aligned}
$$

with the limiting values

$$
\begin{aligned}
G_{Z}(0, x) & =-\frac{x}{2(1-x)} \ln x \\
F_{\text {Box }}(0, x) & =\frac{4}{1-x}+\frac{4 x}{(1-x)^{2}} \ln x, \quad F_{\text {XBox }}(0, x)=-\frac{1}{1-x}-\frac{x}{(1-x)^{2}} \ln x .
\end{aligned}
$$




\begin{tabular}{|c|ccc|}
\hline Nucleus ${ }_{Z}^{A} \mathrm{~N}$ & $Z_{\text {eff }}$ & $\left|F_{p}\left(-m_{\mu}^{2}\right)\right|$ & $\Gamma_{\text {capt }}\left(10^{6} s^{-1}\right)$ \\
\hline${ }_{13}^{27} \mathrm{Al}$ & 11.5 & 0.64 & 0.7054 \\
${ }_{22}^{48} \mathrm{Ti}$ & 17.6 & 0.54 & 2.59 \\
${ }_{79}^{197} \mathrm{Au}$ & 33.5 & 0.16 & 13.07 \\
${ }_{82}^{208} \mathrm{~Pb}$ & 34.0 & 0.15 & 13.45 \\
\hline
\end{tabular}

Table 2. Nuclear form factors and capture rates.

As a result, eqs. (A.4) and (A.5) are given by

$$
\begin{aligned}
F_{u}(x)= & \frac{1}{72(1-x)^{4}}\left[(1-x) x\left\{27(1-x)^{2}+4 s_{W}^{2}\left(31 x^{2}-76 x+21\right)\right\}\right. \\
& \left.+x\left\{27(2-x)(1-x)^{2}+8 s_{W}^{2}\left(8 x^{3}-11 x^{2}-15 x+6\right)\right\} \ln x\right], \\
F_{d}(x)= & \frac{1}{72(1-x)^{4}}\left[(1-x) x\left\{27(1-x)^{2}-2 s_{W}^{2}\left(31 x^{2}-76 x+21\right)\right\}\right. \\
& \left.+x\left\{27 x(1-x)^{2}-4 s_{W}^{2}\left(8 x^{3}-11 x^{2}-15 x+6\right)\right\} \ln x\right] .
\end{aligned}
$$

Open Access. This article is distributed under the terms of the Creative Commons Attribution License (CC-BY 4.0), which permits any use, distribution and reproduction in any medium, provided the original author(s) and source are credited.

\section{References}

[1] P. Minkowski, $\mu \rightarrow e \gamma$ at a Rate of One Out of $10^{9}$ Muon Decays?, Phys. Lett. B 67 (1977) 421 [INSPIRE].

[2] T. Yanagida, Horizontal Symmetry And Masses Of Neutrinos, Conf. Proc. C 7902131 (1979) 95 [INSPIRE].

[3] M. Gell-Mann, P. Ramond and R. Slansky, Complex Spinors and Unified Theories, Conf. Proc. C 790927 (1979) 315 [arXiv:1306.4669] [InSPIRE].

[4] R.N. Mohapatra and G. Senjanović, Neutrino Mass and Spontaneous Parity Violation, Phys. Rev. Lett. 44 (1980) 912 [INSPIRE].

[5] J. Schechter and J.W.F. Valle, Neutrino Masses in $\mathrm{SU}(2) \times \mathrm{U}(1)$ Theories, Phys. Rev. D 22 (1980) 2227 [INSPIRE].

[6] J. Schechter and J.W.F. Valle, Neutrino Decay and Spontaneous Violation of Lepton Number, Phys. Rev. D 25 (1982) 774 [inSPIRE].

[7] T.A. Mueller et al., Improved Predictions of Reactor Antineutrino Spectra, Phys. Rev. C 83 (2011) 054615 [arXiv: 1101.2663] [INSPIRE].

[8] P. Huber, On the determination of anti-neutrino spectra from nuclear reactors, Phys. Rev. C 84 (2011) 024617 [Erratum ibid. C 85 (2012) 029901] [arXiv:1106.0687] [INSPIRE]. 
[9] G. Mention et al., The Reactor Antineutrino Anomaly, Phys. Rev. D 83 (2011) 073006 [arXiv:1101.2755] [INSPIRE].

[10] LSND collaboration, A. Aguilar-Arevalo et al., Evidence for neutrino oscillations from the observation of $\bar{\nu}_{e}$ appearance in a $\bar{\nu}_{\mu}$ beam, Phys. Rev. D 64 (2001) 112007 [hep-ex/0104049] [INSPIRE].

[11] MiniBoone collaboration, A.A. Aguilar-Arevalo et al., A Search for electron neutrino appearance at the $\Delta m^{2} \sim 1 \mathrm{eV}^{2}$ scale, Phys. Rev. Lett. 98 (2007) 231801 [arXiv:0704.1500] [INSPIRE].

[12] MiniBoonE collaboration, A.A. Aguilar-Arevalo et al., Event Excess in the MiniBooNE Search for $\bar{\nu}_{\mu} \rightarrow \bar{\nu}_{e}$ Oscillations, Phys. Rev. Lett. 105 (2010) 181801 [arXiv:1007.1150] [INSPIRE].

[13] MiniBoonE collaboration, A.A. Aguilar-Arevalo et al., Improved Search for $\bar{\nu}_{\mu} \rightarrow \bar{\nu}_{e}$ Oscillations in the MiniBooNE Experiment, Phys. Rev. Lett. 110 (2013) 161801 [arXiv:1207.4809] [arXiv:1303.2588] [INSPIRE].

[14] M.A. Acero, C. Giunti and M. Laveder, Limits on $\nu_{e}$ and $\bar{\nu}_{e}$ disappearance from Gallium and reactor experiments, Phys. Rev. D 78 (2008) 073009 [arXiv:0711.4222] [InSPIRE].

[15] C. Giunti and M. Laveder, Statistical Significance of the Gallium Anomaly, Phys. Rev. C 83 (2011) 065504 [arXiv: 1006.3244] [INSPIRE].

[16] M. Drewes, The Phenomenology of Right Handed Neutrinos, Int. J. Mod. Phys. E 22 (2013) 1330019 [arXiv: 1303.6912] [INSPIRE].

[17] M. Fukugita and T. Yanagida, Baryogenesis Without Grand Unification, Phys. Lett. B 174 (1986) 45 [INSPIRE].

[18] KamLAnD-Zen collaboration, A. Gando et al., Search for Majorana Neutrinos near the Inverted Mass Hierarchy Region with KamLAND-Zen, Phys. Rev. Lett. 117 (2016) 082503 [arXiv: 1605. 02889] [INSPIRE].

[19] G. 't Hooft, Naturalness, chiral symmetry, and spontaneous chiral symmetry breaking, NATO Sci. Ser. B 59 (1980) 135 [hep-ph/9411281] [InSPIRE].

[20] D. Wyler and L. Wolfenstein, Massless Neutrinos in Left-Right Symmetric Models, Nucl. Phys. B 218 (1983) 205 [inSPIRE].

[21] R.N. Mohapatra and J.W.F. Valle, Neutrino Mass and Baryon Number Nonconservation in Superstring Models, Phys. Rev. D 34 (1986) 1642 [InSPIRE].

[22] M.C. Gonzalez-Garcia and J.W.F. Valle, Fast Decaying Neutrinos and Observable Flavor Violation in a New Class of Majoron Models, Phys. Lett. B 216 (1989) 360 [InSPIRE].

[23] A. Atre, T. Han, S. Pascoli and B. Zhang, The Search for Heavy Majorana Neutrinos, JHEP 05 (2009) 030 [arXiv:0901.3589] [inSPIRE].

[24] M. Drewes and B. Garbrecht, Experimental and cosmological constraints on heavy neutrinos, arXiv:1502.00477 [INSPIRE].

[25] P.S.B. Dev, A. Pilaftsis and U.-k. Yang, New Production Mechanism for Heavy Neutrinos at the LHC, Phys. Rev. Lett. 112 (2014) 081801 [arXiv: 1308.2209] [InSPIRE].

[26] A. Das, P.S. Bhupal Dev and N. Okada, Direct bounds on electroweak scale pseudo-Dirac neutrinos from $\sqrt{s}=8 \mathrm{TeV}$ LHC data, Phys. Lett. B 735 (2014) 364 [arXiv:1405.0177] [INSPIRE]. 
[27] A. Das and N. Okada, Improved bounds on the heavy neutrino productions at the LHC, Phys. Rev. D 93 (2016) 033003 [arXiv: 1510.04790] [InSPIRE].

[28] A.M. Baldini et al., MEG Upgrade Proposal, arXiv:1301.7225 [INSPIRE].

[29] A. Blondel et al., Research Proposal for an Experiment to Search for the Decay $\mu \rightarrow$ eee, arXiv: 1301.6113 [INSPIRE].

[30] R.J. Barlow, The PRISM/PRIME project, Nucl. Phys. Proc. Suppl. 218 (2011) 44 [inSPIRE].

[31] C.-Y. Chen and P.S.B. Dev, Multi-Lepton Collider Signatures of Heavy Dirac and Majorana Neutrinos, Phys. Rev. D 85 (2012) 093018 [arXiv:1112.6419] [INSPIRE].

[32] A. Das and N. Okada, Inverse seesaw neutrino signatures at the LHC and ILC, Phys. Rev. D 88 (2013) 113001 [arXiv: 1207.3734] [INSPIRE].

[33] E. Arganda, M.J. Herrero, X. Marcano and C. Weiland, Imprints of massive inverse seesaw model neutrinos in lepton flavor violating Higgs boson decays, Phys. Rev. D 91 (2015) 015001 [arXiv: 1405.4300] [INSPIRE].

[34] S. Antusch and O. Fischer, Non-unitarity of the leptonic mixing matrix: Present bounds and future sensitivities, JHEP 10 (2014) 094 [arXiv: 1407.6607] [INSPIRE].

[35] A. Abada, V. De Romeri, S. Monteil, J. Orloff and A.M. Teixeira, Indirect searches for sterile neutrinos at a high-luminosity Z-factory, JHEP 04 (2015) 051 [arXiv:1412.6322] [INSPIRE].

[36] F.F. Deppisch, P.S. Bhupal Dev and A. Pilaftsis, Neutrinos and Collider Physics, New J. Phys. 17 (2015) 075019 [arXiv: 1502.06541] [INSPIRE].

[37] S. Antusch and O. Fischer, Testing sterile neutrino extensions of the Standard Model at future lepton colliders, JHEP 05 (2015) 053 [arXiv: 1502.05915] [INSPIRE].

[38] E. Arganda, M.J. Herrero, X. Marcano and C. Weiland, Exotic $\mu \tau j j$ events from heavy ISS neutrinos at the LHC, Phys. Lett. B 752 (2016) 46 [arXiv:1508.05074] [INSPIRE].

[39] A. Das, P. Konar and S. Majhi, Production of Heavy neutrino in next-to-leading order QCD at the LHC and beyond, JHEP 06 (2016) 019 [arXiv: 1604.00608] [INSPIRE].

[40] S. Antusch, E. Cazzato and O. Fischer, Displaced vertex searches for sterile neutrinos at future lepton colliders, arXiv: 1604.02420 [INSPIRE].

[41] T. Golling et al., Physics at a $100 \mathrm{TeV}$ pp collider: beyond the Standard Model phenomena, arXiv: 1606.00947 [INSPIRE].

[42] V. De Romeri, M.J. Herrero, X. Marcano and F. Scarcella, Lepton flavor violating Z decays: A promising window to low scale seesaw neutrinos, arXiv:1607.05257 [INSPIRE].

[43] S.S.C. Law and K.L. McDonald, Generalized inverse seesaw mechanisms, Phys. Rev. D 87 (2013) 113003 [arXiv:1303.4887] [INSPIRE].

[44] J. Lopez-Pavon, S. Pascoli and C.-f. Wong, Can heavy neutrinos dominate neutrinoless double beta decay?, Phys. Rev. D 87 (2013) 093007 [arXiv: 1209.5342] [INSPIRE].

[45] P.S.B. Dev and A. Pilaftsis, Minimal Radiative Neutrino Mass Mechanism for Inverse Seesaw Models, Phys. Rev. D 86 (2012) 113001 [arXiv:1209.4051] [InSPIRE].

[46] P.S. Bhupal Dev and A. Pilaftsis, Light and Superlight Sterile Neutrinos in the Minimal Radiative Inverse Seesaw Model, Phys. Rev. D 87 (2013) 053007 [arXiv:1212.3808] [INSPIRE]. 
[47] Z. Maki, M. Nakagawa and S. Sakata, Remarks on the unified model of elementary particles, Prog. Theor. Phys. 28 (1962) 870 [InSPIRE].

[48] B. Pontecorvo, Neutrino Experiments and the Problem of Conservation of Leptonic Charge, Sov. Phys. JETP 26 (1968) 984 [Zh. Eksp. Teor. Fiz. 53 (1967) 1717] [InSPIRE].

[49] J.A. Casas and A. Ibarra, Oscillating neutrinos and $\mu \rightarrow e, \gamma$, Nucl. Phys. B 618 (2001) 171 [hep-ph/0103065] [INSPIRE].

[50] E.K. Akhmedov, V.A. Rubakov and A.Yu. Smirnov, Baryogenesis via neutrino oscillations, Phys. Rev. Lett. 81 (1998) 1359 [hep-ph/9803255] [INSPIRE].

[51] A. Abada, G. Arcadi, V. Domcke and M. Lucente, Lepton number violation as a key to low-scale leptogenesis, JCAP 11 (2015) 041 [arXiv:1507.06215] [INSPIRE].

[52] J. Kopp, P.A.N. Machado, M. Maltoni and T. Schwetz, Sterile Neutrino Oscillations: The Global Picture, JHEP 05 (2013) 050 [arXiv: 1303.3011] [InSPIRE].

[53] A. Abada, G. Arcadi and M. Lucente, Dark Matter in the minimal Inverse Seesaw mechanism, JCAP 10 (2014) 001 [arXiv:1406.6556] [INSPIRE].

[54] A. Abada and M. Lucente, Looking for the minimal inverse seesaw realisation, Nucl. Phys. B 885 (2014) 651 [arXiv:1401.1507] [INSPIRE].

[55] A. Abada, V. De Romeri and A.M. Teixeira, Effect of steriles states on lepton magnetic moments and neutrinoless double beta decay, JHEP 09 (2014) 074 [arXiv:1406.6978] [INSPIRE].

[56] E. Fernandez-Martinez, J. Hernandez-Garcia and J. Lopez-Pavon, Global constraints on heavy neutrino mixing, JHEP 08 (2016) 033 [arXiv:1605.08774] [INSPIRE].

[57] M.C. Gonzalez-Garcia, M. Maltoni and T. Schwetz, Updated fit to three neutrino mixing: status of leptonic CP violation, JHEP 11 (2014) 052 [arXiv:1409.5439] [INSPIRE].

[58] Planck collaboration, P.A.R. Ade et al., Planck 2015 results. XIII. Cosmological parameters, Astron. Astrophys. 594 (2016) A13 [arXiv: 1502.01589] [INSPIRE].

[59] L. Delle Rose, C. Marzo and A. Urbano, On the stability of the electroweak vacuum in the presence of low-scale seesaw models, JHEP 12 (2015) 050 [arXiv:1506.03360] [INSPIRE].

[60] A. Abada, V. De Romeri and A.M. Teixeira, Impact of sterile neutrinos on nuclear-assisted cLFV processes, JHEP 02 (2016) 083 [arXiv: 1510.06657] [INSPIRE].

[61] SINDRUM II collaboration, C. Dohmen et al., Test of lepton flavor conservation in $\mu \rightarrow e$ conversion on titanium, Phys. Lett. B 317 (1993) 631 [INSPIRE].

[62] L. Canetti, M. Drewes, T. Frossard and M. Shaposhnikov, Dark Matter, Baryogenesis and Neutrino Oscillations from Right Handed Neutrinos, Phys. Rev. D 87 (2013) 093006 [arXiv: 1208.4607] [INSPIRE].

[63] R. Alonso, M. Dhen, M.B. Gavela and T. Hambye, Muon conversion to electron in nuclei in type-I seesaw models, JHEP 01 (2013) 118 [arXiv: 1209.2679] [INSPIRE].

[64] A. Abada, M.E. Krauss, W. Porod, F. Staub, A. Vicente and C. Weiland, Lepton flavor violation in low-scale seesaw models: SUSY and non-SUSY contributions, JHEP 11 (2014) 048 [arXiv: 1408.0138] [INSPIRE]. 